\title{
The Effect of Anarchistic Actions in Digital Product Innovation Networks: The Case of "Over the Air" Software Updates
}

\author{
Vincent Isaksson \\ vincent.isaksson@vincea.se \\ Services Advisor
}

\author{
Lena Hylving \\ lena.hylving@viktoria.se \\ Viktoria Swedish ICT
}

\begin{abstract}
In this paper we explore mirroring challenges when an incumbent firm endeavor digital innovation. More specifically, we describe how AutoInc, organized according to the physical vehicle it produces, is challenged when an "over the air" software service is developed and implemented.

Using the mirroring hypothesis as a point of departure to understand existing and emerging innovation networks, we recognize anarchistic actions. The analysis reveals the emergence of anarchic actions and how they challenge wellestablished federative innovation networks within the organization. With continued focus on technology, the project and organization disregarded necessary social structure development, which resulted in reduced capabilities to utilize the digitalized service.

This qualitative paper also illustrates how the mirroring hypothesis, although originating from product innovation literature, can be used to understand digitalization dynamics. To the end, the analysis shows that the digital product innovation classification structure may need additional tuning.
\end{abstract}

\section{Introduction}

Incumbent firms are challenged today due to digitalization [1-4]. For example, the traditional camera industry was seriously challenged when digital imaging disrupted established business models and organizing $[5,6]$ and it has been recognized that social structures, such as processes and skills, needs an update in order to cope with the increase of software in cars [7]. Not coping with the social structures connected to technology advancement has proven potentially disastrous [6]. However, it has also been highlighted how digital innovation together with institutional entrepreneurship has proven successful in incumbent firms [8].

In the last couple of years, much research has focused on digital innovation exploring the underlying mechanisms, inbuilt practices associated with digital innovation and challenges and possibilities connected to it [9-11]. In this paper we continue on this path and explore digital product innovation networks [12] within a firm. More so, we use the classification of digital product innovation networks as sensitizing concepts [13] in order to understand what happens in organizations when digitalized solutions are developed and introduced into an incumbent firm fitted for product manufacturing. We have studied an automotive manufacturer, AutoInc, with a particular focus on their development work enabling Over-The-Air (OTA) software updates. In order to understand the prerequisites for the organization to undertake the OTA service and do digital innovation, we used the mirroring hypotheses [14] as a foundation for our analysis as the mirroring concept is reliably applicable to automotive organizational settings.

Mirroring hypothesis stems from modularity, product innovation and manufacturing, arguing that an organization should mirror the product it produces in order to be successful $[15,16]$. AutoInc, as many incumbent product developing firms, was organized according to its physical components, such as a division dedicated to powertrain (engine) and one to chassis (the internal physical frame). However, with digitalization and digital innovation, such mirroring needs to be rethought and adjusted [9].

With this as a background, we asked ourselves: How does digital innovation influence mirroring in incumbent firms? And more so, how can we understand the effects of different digital product innovation networks within the same organization?

With empirical data collected during a period of three years at AutoInc, we show how different digital product innovation networks exists within the same firm and what effects that have. Overall, this study generates the following insights:

- Anarchic actions, in the federative innovation network can cause disturbance and responsibility confusion. 
- Anarchy can be interpreted differently (as cooperative or non-cooperative) and be manifested in at least two different ways; i) directly by dictating business-goals and ii) indirectly by dictating what fundamental technological capabilities would be developed and thereby the possible solutions that in turn limit or dictates possible business.

- Practical applicability of the mirroring hypothesis can assist in overcoming digitalization dynamics.

The paper proceeds as follows; we begin by reviewing the mirroring hypothesis $[14,17]$ as we can see strong support for mirroring at AutoInc. This is followed by an overview of the digital innovation literature. In the digital innovation literature we hone in on digital product innovation networks, which we use as sensitizing concepts [13] in order to understand what was going on at AutoInc. We further direct our attention to anarchism $[18,35]$ and shortly give an overview of the concept. We then describe the method used when collecting and analyzing the empirical data, followed by presenting the empirical case and insights. We end the paper with a discussion of our insights and finalize the paper with our conclusions.

\section{The mirroring hypothesis}

The mirroring hypothesis literature is based on modularity and asserts that organizational structures corresponds to the technical architecture the organization attempts to develop. The mirroring is described as linkages between the design and tasks required in order to realize the design $[14,17]$. Other research call this socio-technical congruence [19], fundamental isomorphism [16], or plainly morphisms [1].

Research has shown that mirroring is highly valuable when understanding why organizations are structured the way they are dependent on what they are developing and producing. It has also been highlighted that the mirroring hypothesis does not apply well to open collaborative projects, most of which focused on software development [17]. However, researchers have used the theory as a way to understand what happens to organizations when digitalization dynamics trigger changes, for example when new digital tools are taken into use or new software based processes are required $[1,20]$.

Hylving [1] extends the concept of mirroring, or morphisms, to include second order morphisms. The second order morphisms recognize how other structures indirectly are connected to the artifact being developed. For example, developing user experience (UX) possibilities, instead of only a physical product, require new business models. However, when developing the UX solution it is easily done to only focus on the UX solution itself, and forget about what supporting structures are needed in order to utilize the UX solution [1]. In other words, it describes how everything is interconnected to each other in one or the other way.

In this paper we use mirroring hypothesis as a starting point since the empirical setting is a product manufacturing firm with well-established organizational structures based on the physical architecture of the product they produce; the vehicle.

\section{Digital innovation and digital product innovation networks}

Digital innovation can be defined as the carrying out of new combinations of digital and physical components to produce novel products [9]. Digital artifacts are produced by ever-changing combinations of patterns of technologies, routines and cognition [21]. This offers new ways of interacting with, thinking about, relating to and working with the developed artifacts [22]. As many different industries are going thru digital transformations endeavoring digital innovation, new logics emerge [2]. For example, the automotive industry traditionally considered as a manufacturing industry producing vehicles, now is categorized as a platform industry $[8,23,24]$ where a federative way of working is implemented around the platform as the central module in the highly modularized product architecture. Naturally, this involves the network of actors responsible for those modules.

Based on the idea of a network of heterogeneous actors [25, 26], digital product innovation networks is defined as groups of more or less heterogeneous actors and their tools. These groups are included in complex socio-technical networks that embody different forms of knowledge [12]. Digital product innovation is thereby a distributed phenomenon characterized by network effects, messiness, ambiguity, and combinability [21].

There are four different digital product innovation networks, namely; Project, Clan, Federated and Anarchic [12]. The Project innovation network consists of a rather homogenous group of people where control and coordination is centralized. The Clan also consists of homogenous people but the coordination and control is distributed. The Clan is usually driven by a common interest and relatively well-defined set of tools that are readily identified 
and mobilized for effective cognitive and social translations [12]. Example of a clan network are the open-source communities [27, 28] where the actors act according to their common goal, but with very limited, or no, centralized control and coordination.

The federated innovation network has centralized controlled but uses knowledge from a diverse set of resources. In large manufacturing organizations, such as automotive OEMs, federated innovation networks can be considered norm as knowledge, expertise and skills are distributed although the control and coordination of these resources are centralized [8].

The Digital Innovation definition of anarchic action is based on the dimension of control of the process, structure and outcomes, which is distributed (and not centrally governed) [12]. This definition also includes knowledge resources being distributed and not being known a priori, as exemplified in [36]. According to the Meriam-Webster Dictionary [35], anarchism in general is defined as absence or denial of any authority or established order. Others have described the ultimate goal of anarchism as being to create a free society which allows all human beings to realize their full potential [18] and to deny authority [29]. The general definition of anarchism is thereby relative to a presumed governance of some form while digital innovation essentially defines it as any action that is self-motivated, albeit still cooperative. Anarchic innovation networks are in this sense dynamic and complex socio-technical systems consisting of heterogeneous and self-driven actors operating a diverse set of tools and other resources. [12]. This diversity of control makes different innovation trajectories intervene with and influence each other [8]. Anarchic action is here defined as action taken by actors in anarchic digital innovation networks.

Institutional entrepreneurship [30] and anarchic action [12] are significantly but not entirely overlapping ways of acting and being. Institutional entrepreneurship is for instance inherently endogenous and relative to some form of governance [30] which anarchic actions need not be as such restrictions are not present in that definition [12]. On the other hand, anarchic actions stipulate distributed knowledge resources [12] whereas the definition of institutional entrepreneurship does not specify such a restriction [30]. Institutional entrepreneurs are organized actors who envision new institutions as a means of advancing interests they value highly yet that are suppressed by extant logics [30]. Institutional entrepreneurs, thus, clearly fulfill the basic requirements to be considered anarchic as defined by [12]. However, Institutional entrepreneur's actions are on top of this also aware, and calculative from a positive sense (i.e. cooperative and constructive) [30]. They are often less connected to organizational norms and have a weaker connection to established processes and also that they are often disadvantaged by prevailing arrangements and stand to benefit from change [31]. This presumption of drivers behind the action does not exist in the definition of anarchic action. This however raises the question what is meant by negative, destructive contribution or passiveness and what anarchic label such action then should have and also who should make the determination of what is positive or negative.

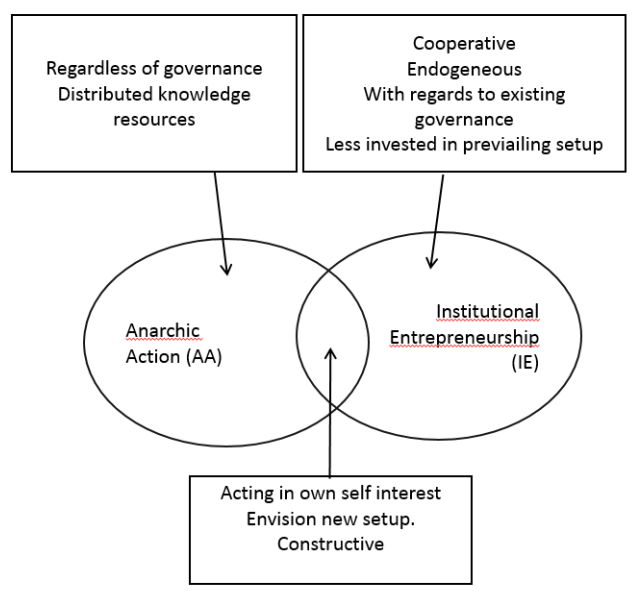

\section{Figure 1: Institutional entrepreneurship vs. Anarchic action}

Consequently, there is a void in the more detailed definition of institutional entrepreneurship and less so in the less restricted (and less colorful) definition of action in anarchic digital innovation networks [12].

\section{Method}

The following section describes the methodological approach on this qualitative paper.

\subsection{Data Collection}

Along the development cycle of the OTA software service, the main author participated in the development and observed the progress. This allowed him to take part of all business-, requirements- and project meetings along with having access to documentation connected to the project. The participation of the first author started with the industrialization of the function in year 2012 and ended with the quality audit of the project in year 2015. Data were collected under a non-disclosure agreement and include several hundred documents. 
For this paper, we focused on project-, governance- and requirements-documents in the form of emails, minutes-of-meetings, generated office documents, diary notations, plans, and presentations. Data was also collected in interviews that were partly transcribed and anonymized before being brought into the data analysis.

\subsection{Data analysis}

The analysis of the case study- and project writeups [32] were run through key participants in order ensure its correctness. Analysis was carried out jointly in the research team to achieve complementary insights and enhanced confidence in the findings [33]. The write-ups as well as interview transcripts were the continuous basis of a thematic and inductive analysis [37, 13] that focused on identifying inconsistencies in the three aspects of Organization/Process, Project and Functionality. The focus was to detect 'problems' which were then elaborated and categorized and screened through discussions regarding distinctiveness, involved kinds of structures ('is it a lack in social-to-technical mirroring, in social-to-social or something else?') and also regarding usability in research documentation and presentations.

\section{Empirical Findings}

\subsection{AutoInc before and during the project}

Ever since the start of the European vehicle manufacturer AutoInc, the company has been organized around the physical architecture of the vehicle and its traditional lifecycle; design, production, sales, and maintenance. Overall, the company has had a Product Planning (PP) department ordering vehicles and other products, an $R \& D$ department designing the vehicles, and Manufacturing $(\mathrm{M} / \mathrm{F})$ responsible for turning those designs into real vehicles. Manufactured vehicles have then been made available for sales by the Marketing and Sales (MS) organization and then the After Sales (AS) organization has provided Dealers $\&$ Workshops with the tools and parts to service the vehicles on behalf of the vehicle owners. Finally, an IT department providing all of the company with computers to automate the work as well as a Financial (FI) department assuring the money needed to sustain all these activities.

In particular R\&D, Purchasing and Manufacturing have internally been organized around the structure of the vehicle. For instance, there has been a group within $\mathrm{R} \& \mathrm{D}$ designing wheels (rims) and a team in purchasing procuring wheels for Manufacturing who have had a team responsible for preparing wheels for manufacturing and a station in the production line where wheels were added to the vehicles being manufactured.

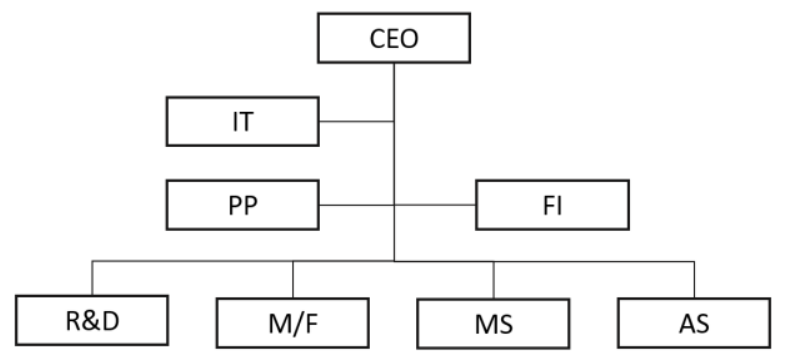

Figure 2: Autolnc Organization

Over the decades of vehicle evolution, as vehicles begun having electrical system, there has likewise been departments throughout the company managing what started out as simple lights and horn to today's advanced IT networks on wheels. A car from AutoInc include more than 100 small computers (for the vehicle alone) called ECUs - Electrical Control Units. Each ECU has their own set of software's programmed into them to do their respective parts of the necessary functionalities, for example controlling breaks or handling all that is visible in the main infotainment display. The organization of the electrical department in R\&D has evolved with this evolution in the structure of electrical systems or functionalities, including software development teams for areas such as energy management, infotainment and safety. Consequently, a significant flow of software maintenance updates and patches (software parts) have also been created, tested, approved and provided to the vehicle workshops for installation as part of the traditional vehicle service appointments. All in very much the same way as hardware parts.

\subsection{Digitalization transformations at AutoInc}

In 2012, inspired by the smartphone mobile industry and another OEM, AutoInc embarked on an infotainment platform project. The infotainment system was going to be based on apps such as Spotify and ParkingFinder working on top of an ordinary operating system instead of having all functionality written into one big software embedded in a physical component. This would allow fast changes and innovation to customer-centric functionalities while still also having a more stable basic set of car-centric functionalities. It would also reduce cost since the 
operating system would be something like Linux or Windows instead of some proprietary and very special software foundation. In this way, the infotainment system could be relatively long-lived, compared to the old concept of a vehicle's life cycle of 5-7 years where software and functionalities were intertwined with hardware and more difficult to evolve after manufacturing.

And so it was decided that a 'remote software update' function, eventually named 'Over The Air' (OTA) software update, was to be created. An R\&D manager expressed it like this:

"We need to have [vehicle] systems that are up to date. There is a cost in not doing this, but we cannot calculate all the option values. We can't calculate ROI by using OTA during the first 5 years but hereafter it will have a business opportunity. We know however that we can't wait. In such case we will lose sales and get lower customer satisfaction. In the end this is the right thing to do for AutoInc."

The order from the PP organization was to allow the customer in the vehicle to use a set of preloaded and continuously updated apps, and also to update any of the basic system software of all of the ECUs. The order prioritized the handful of ECU's that were going to be involved in Internet connection capabilities and thereby suffer risk of hacking. This first order for OTA function therefore also included possibilities to update Bluetooth security, WiFi security, and Infotainment graphics.

To match the new situation, the electrical department within R\&D organized an Infotainment Platform group responsible for vehicle Applications Programming Interfaces (APIs). The API's would allow user applications like Spotify and Android Auto to access less risky vehicle functionalities and data like fan speed settings, temperature level, speed information, fuel level and more. A group called Connectivity was also established. They were responsible for the realization of all Internet connection related solutions (such as Bluetooth, WiFi, Mobile tethering, 3G/4G).

R\&D also initiated an Advanced Engineering predevelopment project, an 'AE', with the intent to deliver technical concepts for the OTA function, for example software packaging formats, download- and installation mechanisms and security solutions. The time plan was to be part of the new vehicle to be launched in 2015. The AE-project considered OTA as "just another channel" - a wireless version of the cord-based software download system that was used to service the software of the vehicles in vehicle workshops. But instead of this hardwired cord, the OTA technology would use mobile communication technology $(3 \mathrm{G} / 4 \mathrm{G})$ and a cloud platform to transfer the software to the vehicle, and a system in the vehicle would do the software updates of all the ECU's in the vehicle.

In Q3 2012, a Function Owner (FO) was added to the OTA project. The responsibility of the FO was to design the function and in this also translate businessrequirements on the OTA function into function requirements, specifying what and how the OTA function should work on a solution-independent level.

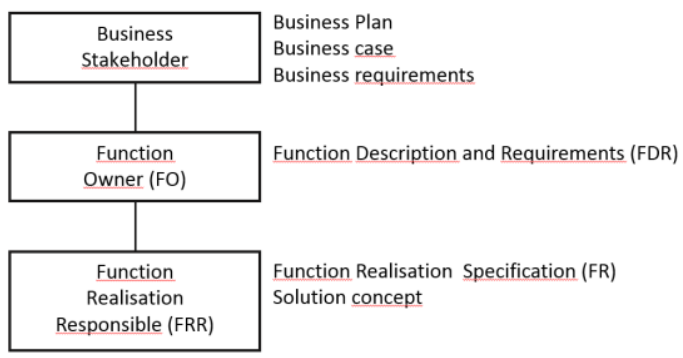

Figure 3: Requirements Organization

For instance, a business requirement like "The function must be robust" could be translated into functional requirements like:

a) "The data and information sent and received by the function must be handled by robust protocols so that no data is lost due to more or less sporadic Internet connection between the vehicle and AutoInc",

and performance requirements such as:

b) "The function must take less than 5 seconds in continuing operations after downtime or loss of connectivity".

All such functional and performance requirements were documented in the FDR (Function Description and Requirements) document. The leader of the AE project was subsequently appointed as the Function Realization Responsible (FRR) with the task to design a solution and in this translate the FDR specification into a Function Realization specification (FR) and drive creation of a solution that would fit that FR. For instance, the requirement a) presented above was translated into solution requirements such as "There must be a cached communication between vehicles and servers of the following format ..." or "All communication must contain error-detection and -correction data of this kind ...".

At this time, the AE-project had already developed the solution concept and the FR was mostly already expressing requirements to suppliers and also to adjacent technological areas in the car in line with that pre-developed concept. Consequently, a substantial discrepancy was eventually identified 
between the function specification (FDR) and the Function realization specification (FR).

The initial dialogs between FO and different business stakeholders, such as MS and AS, also revealed the absence of business requirements on the function (what kind of customers will use this function and how - what do they want?) and overall business model (how do we produce and sell this?) as well as use-cases (how should the function be used?). Essentially, it became apparent that the role of Business Stakeholder or business owner never had been formally defined at AutoInc since the business had always been about selling vehicles and accessories, not services or software.

It also became clear that the organization did not have the processes or people to enter into continuous dialog with actual customers and from this express formal business plans and requirements. Instead, customer input was mostly gained through 3rd party market reports and occasional interview studies. In addition, it became clear that engineers and project managers had compensated the lack of business owners and their input requirements and calculations by creating rudimentary business-cases themselves to motivate or eject deliverables to/from the projects. In addition, the business focus of the engineers was on vehicle cost. Lifecycle aspects of the deliverables as well as profit or even revenue, was limited.

In Q1 2013, R\&D surprised the company when one of its officials stated: "We will be allowing the customer to add apps individually per vehicle". This new direction was contrary to the already decided path by PP and the FO together that the OTA function would just update a standard set of preinstalled systems software, apps and other features per market. With this additional capability (customer being able to individually add functionality per vehicle), the complexity of the function increased and new significant risks were added to the project. For example, risks associated with having on-demand variety in apps, risk of slow/faulty or absent downloads. Additional risks related to handling of content per vehicle, keeping track of actual individual status of software in the vehicles, timing- and errorsensitivity aspects as well as a higher demand on customer interaction. This more difficult goal also drove new requirements on testing, training, documentation and marketing.

Finally, the new direction turned the function into a highly customer-centric function as compared to car-centric functionality. This meant that the organization to produce more customer-centric functionalities such as 'Restplaces' or 'StoryTel', was going to need to grow and become more productive and effective, extrovert and agile than before the scope change.

R\&D needed the function to work as a means to alleviate pressure on the product development process (software quality instead could be complemented during shipment of car to dealers). The function also enabled $R \& D$ to regularly patch internet related security software to latest possible status. Finally, it also meant a possibility to regularly offer new apps in the infotainment system between new and old vehicle programs. To assure these benefits, R\&D claimed the role of business-owners and thereby the entitlement to add to, or change, the scope of the original order. Although the new demands articulated by R\&D added cost, complexity and risk the whole vehicle project, the strong legitimacy of R\&D within AutoInc, gave them the power to dictate what would be delivered or not.

In Q2 2013, a Project Manager (PM) for the OTA project as sub-project to the total vehicle project, was appointed at the AS organization. The PM started weekly cross-organizational meetings where all technical coordination was brought up. All technical leaders related to the in-vehicle solution as well as outside of vehicle ('off-board'), were invited. The weekly meetings focused on different technological components of the OTA solution, and actions required to manage overall risks of not delivering the OTA function with right quality in time.

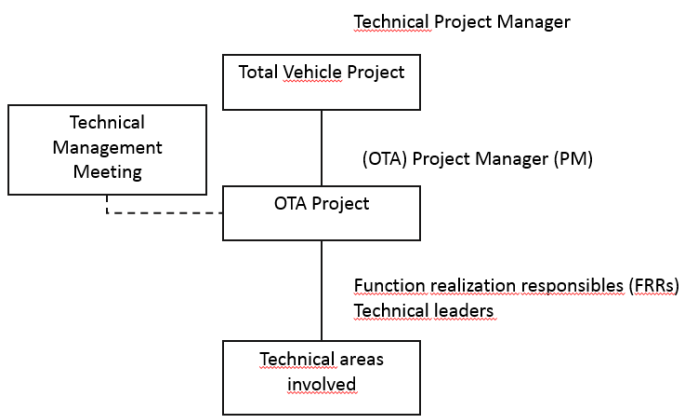

Figure 4: Governance Organization

On an overall OTA project governance level, a weekly governing Technical Management Meeting was launched, involving senior line managers from R\&D. This way of organizing sub-project was standard operations in vehicle development projects. Through technical management meetings like the one for OTA, AS, IT and MS together with R\&D jointly handled overall governing decisions regarding resources and priorities of all major separate projects.

At this time the FRR informed the project that R\&D would focus on 'the OTA channel' because this was the vehicle-centric part of the solution. This basically meant that the technical solution needed for 
the OTA function in the vehicle was essentially the only priority for $R \& D$. This was in line with the prevailing Automotive development view and business model focusing on car and technology. As an incumbent traditional vehicle development organization, R\&D would not take responsibility for technical requirements or development outside the vehicle that was not directly and technically linked to the vehicle (such as supporting IT, sales support, workshop support), nor for operations development (adding staff, creating or changing processes), content handling (overall managing what functionalities were built into what software) nor for serviceability (assuring solutions for the vehicle workshops to be able to service the vehicle). The governing Technical Management Meeting accepted this.

In Q1 2014, R\&D communicated that due to technical limitations, the current software (that was traditionally developed to be installed in the vehicle in the factory and to update and maintain the vehicles at workshops), was not automatically going to be possible to install via the OTA function. Any software intended for deployment to vehicles via the OTA function had to be specifically tailored and packaged. If any part of the OTA-package included a software that the specific part of the vehicle did not support being installed via OTA, then none of the software in that package would become installed. Since there were only a few parts of the vehicles that were going to be able to support OTA software installation, this restriction considerably limited the potential use of the function. Especially since software packages would almost always contain multiple software parts. Neither the Technical Management Meeting nor the vehicle project addressed this issue. Consequently, the project organization was never complemented to accommodate for the need of generating a new or improved software packaging organization.

In Q2 2014, the persons acting FO and PM were additionally tasked with assuring that the OTA function could be supported worldwide at dealers, in workshops and in call-centers. This included building necessary IT-system for the workshop technicians to use when looking into the potential errors reported by the customers and workshops. The new more customer-oriented focus dictated by $R \& D$ here presented new challenges to AS on top of the traditional one;

a) Support and fault-tracing of a customer-centric function rather than a traditional vehicle-centric function.

b) Support and fault-trace any problems with the explicit content offers that was now going to be individually selectable by end customers and per vehicle.

c) Support a distributed function that was not only implemented in the car but involved the AutoInc Cloud and other systems.

While these consequential challenges were understood by some of the project members, the Technical Management Meeting did not formally include them into the OTA project scope. Nor did the AS organization traditionally have the formal task to define or deploy any separate project for such customer-oriented deliverables. AS traditional task had always been to develop workshop support capability for vehicle-centric functions only. With limited resources, AS never created the capability necessary for customer support and due to lack of resources, the capability for workshop support was also severely limited, even by traditional measures.

In Q3 2015 a quality audit following the official end of the project recognized, elicited and documented 55 problems from the OTA project. This included 42 issues pertaining to governance and project management, 9 pertaining to development process and actual realization of the function and 2 issues related to organization and processes. Overall, the audit summarized that the limited managerial as well as technical and social understanding of the sociotechnical structure and complexity of the deliverable, was a factor severely impeding development as well as governance of the development. The report stated that "Responsibility for development of connected functions is partial/unclear". Out of these 55 problems, four (4) represented formal quality system deviations, which were processed by AutoInc managerial teams on behalf of the corporate quality organization.

\section{Discussion}

Reviewed literature, for instance $[1,14,15,16$, 17, 19] all testify to mirroring being more or less pronounced and relevant in development. In our empirics and theoretical study, we have used the term Mismatch to indicate a lack or absence of mirroring and from our empirics, we have excavated 3 exemplifying mismatches.

The first excavated mismatch exemplify how Advanced Engineering can limit delivery of functionality. We saw in the case how the money for the OTA function was already consumed by R\&D advanced engineering. As a result, when the specification for the OTA function was delivered, there was no money nor any time to develop a fitting technology concept. The consequential problem of 
discrepancy between function requirements and this solution concept was solved by truncating the specification to match the pre-developed technology. This essentially limited the possibilities to deliver the OTA solution and required that business accepted delaying the development of the missing functionalities. We find no distinct account for this kind of tech-to-tech mismatches or conflict in the reviewed literature [14, 17].

The second excavated mismatch concerned a dissonance between R\&D and the product planning (PP) regarding the scope of the deliverable as required by business, where $R \& D$ declared that they would extend the solution to include user's ability to add and delete apps in the vehicles. In other words, a change in technological scope or structure posing mismatch with mainly organizational structures. Interestingly, $R \& D$ diversion from the original business order in technical solution design decisions, was common due to $\mathrm{R} \& \mathrm{D}$ leading projects that ultimately had to deliver. With limited resources and a set deadline, quality or size of deliverables basically remained the only variable. Thus, project downscoping and technical compromises are commonplace in the everyday R\&D activity. Open controversy between what in federative sense is the ordering unit (PP) versus delivering unit $(\mathrm{R} \& \mathrm{D})$, was on the other hand rare.

Both this second excavated mismatch and the first were due to anarchic actions as R\&D in both cases acted on its own accord as a separate party outside of the federative norm. In the second example, R\&D not only claimed their traditional jurisdiction over technology solutions but also a partially overriding role on the business ordering level. The motivation was their own need of the service and also being the organization that initiated the idea. R\&D decisions need not necessarily bring additional mirroring needs. However, this second decision redirected development towards additional operational changes.

Regardless of an initiating action being anarchic or not, direct and heterogenic mismatches like the second excavated mismatch, are relatively common in the field of digital innovation within Automotive since the organizational structures are very much aligned with vehicle lifecycle and technology hierarchies and thus in general very poorly resembles the structure of distributed and more horizontally integrated digital services. Such mismatches pose question-marks to the company and projects on who (what organization) should be responsible for some, often entirely new kind of deliverable.

Finally, in the third excavated mismatch no one was assigned from $R \& D$ to be in charge of assuring the process and organization for specifically creating, packaging, testing and release of software that was to be offered and deployed using OTA. R\&D task in the project was to design and deliver the technical OTA solution, not assure there was working content available to use it. The mismatch or lack of mirroring existed regardless of anarchic actions between an organizational need and the already existing $R \& D$ organization tasked with producing and testing all software with the traditional aim of deploying it in factory and in vehicle maintenance only. Since the project never altered its structure to also design necessary organization to produce specific OTA software, ultimately a technical solution was developed but not the organization necessary to use it.

Looking closer to the defining literature $[1,14$, $15,16,17,19]$, we find that neither organization-toorganization nor technology-to-technology mirroring are specifically documented as part of the definition of mirroring. Nor is the relation between cause and effect such as change in technology scope driving a need of other changes or adaptions. This makes it difficult to discuss in terms of what kind of change in one structure would be needed specifically due to a change in another. The theorem and adjacent definitions just define the possible existence of relations between social and technical structures in a timeless manner. Neither does the definitions distinguish between different kinds of technical structures like Communication, HMI (Human Machine Interaction) or Powertrain and how such structures may affect one another. Furthermore, the second order mismatches or propagated lacks of mirroring where one creates another which in turn drives a third (and so on) as defined by [1] as an extension to mirroring, is left without consideration in most other documents.

This study has highlighted the possible existence of mismatches between structures of the same kinds, both social and technical in Automotive digital innovation. The difference in technological structure drove a lack or mirroring in both technology-related organizational structure (more agile and capable software production and customer support) as well as in business organization structures (need to handle software and related information more explicitly as offers portfolio to customers). Likewise, there was a need for difference in company organizational structure that was not mirrored by project organizational structure and thus the company structure was never adjusted. These empirics indicate that the concept of mirroring might be possible to extend further. Finally, the examples of mismatches documented in this article were a few selected from a larger set which required no deeper analysis or 
elicitation to be found. This indicates that there likely are more to be found in this case and possibly in many other practical industrial case.

This study thus indicatively confirms the existence of mirroring and inherent anarchic tendencies within the same otherwise federative Automotive innovation network. The anarchic tendencies exemplified, also provide indicative verification of the predicted "southeastward" movement in the content and scope of product innovation. As also stated by [12], their outlined categories can therefore not be discrete. If, however they are considered additive and scalable properties on a continuum, such a revised theory can better account for the empirics of this study as well as for nuances already found in the nature of (Automotive) digital innovation networks.

Having continuously scaled additive properties however raises the question on how to do a more fine-grained evaluation of innovation network properties. Consequently, scholars aiming to assist in this regard are urged to further explore nuances in the definitions of the grading and definitions of the existing two properties of the product digital network [12] as well as consider if there are additional significant effects deserving to be established as properties. The concept of institutional entrepreneurship [31, 34] offers some possible candidate antecedents to explore further as properties of digital innovation networks. Further dive into these antecedents, into the difference between the concepts and perhaps a suitable basic ontology would be great strides towards a further improved digital innovation networks properties- or classification scheme/continuum.

\section{Conclusion and contribution}

We can draw several conclusions from this paper. First, this paper show that the concept of mirroring is indicatively applicable in manufacturing contexts as well as software development. Secondly, upon entering the world of digital innovation, organizations can suffer from indirect and direct mismatches. That is, we have illustrated how technology does not mirror with technology, organization does not mirror technology, and organization does not mirror organization. Third, anarchic action can exist in federated innovation networks. Consequently, the networks described by Lyytinen et al (2015) are only a foundation for discussion and nothing that can be used to categorize groups/networks of people in a definite way. Thus, it is possible to look at properties of innovation networks and consider the different dimensions as continuum instead of four different categories. Finally, the concept of institutional entrepreneurship may offer candidate antecedents to explore further as properties of anarchic action in digital innovation networks.

\section{References}

[1] Hylving, L., Digitalizations Dynamics: User Interface Innovation in an Automotive Setting, University of Oslo, 2015.

[2] Svahn, F., Mathiassen, L., and Lindgren, R., "Embracing Digital Innovation in Incumbent Firms: How Volvo Cars Managed Competing Concerns", Management Information Systems Quarterly, Forthcoming,

[3] Åkesson, M., Digital Innovation in the Value Networks of Newspapers. Doctoral Dissertation, Chalmers University of Technology \& University of Gothenburg, 2009.

[4] Jonsson, K., Westergren, U., and Holmström, J., "Technologies for Value Creation: An Exploration of Remote Diagnostics Systems in the Manufacturing Industry", Information Systems Journal, 18(3), 2008, pp. 227-246.

[5] Lucas, H., and Goh, J., "Disruptive Technology: How Kodak Missed the Digital Photography Revolution", The Journal of Strategic Information Systems, 18(1), 2009, pp. 46-55.

[6] Tripsas, M., "Technology, Identity, and Inertia through the Lens of "the Digital Photography Company"", Organization Science, 20(2), 2009, pp. 441-460.

[7] Broy, M., "Challenges in Automotive Software Engineering", Proceedings of the 28th international conference on Software engineering, 2006

[8] Henfridsson, O., and Yoo, Y., "The Liminality of Trajectory Shifts in Institutional Entrepreneurship", Organization Science, 25(3), 2013, pp. 932-950.

[9] Yoo, Y., Henfridsson, O., and Lyytinen, K., "The New Organizing Logics of Digital Innovation: An Agenda for Information Systems Research", Information Systems Research, 21(4), 2010, pp. 724-735.

[10] Henfridsson, O., and Bygstad, B., "The Generative Mechanisms of Digital Infrastructure Evolution", MIS quarterly, 37(3), 2013, pp. 907-931.

[11] Fichman, R., Dos Santos, B., and Zheng, Z., "Digital Innovation as a Fundamental and Powerful Concept in the Information Systems Curriculum", Management Information Systems Quarterly, 38(2), 2014, pp. 329-353.

[12] Lyytinen, K., Yoo, Y., and Boland Jr, R.J., "Digital Product Innovation within Four Classes of Innovation Networks", Information Systems Journal, 2015, 
[13] Bowen, G., "Grounded Theory and Sensitizing Concepts", International journal of qualitative methods, 5(3), 2008, pp. 12-23.

[14] Colfer, L., "The Mirroring Hypothesis: Theory and Evidence on the Correspondence between the Structure of Products and Organizations", 2007

[15] Conway, M., "How Do Committees Invent?", Datamation, 14(4), 1968, pp. 28-31.

[16] Baldwin, C., and Clark, K., Design Rules: The Power of Modularity MIT Press, Cambridge, MA, 2000.

[17] Colfer, L., and Baldwin, C., "The Mirroring Hypothesis: Theory, Evidence and Exceptions", Harvard Business School, 2010, pp. 10-058.

[18] Marshall, P., Demanding the Impossible: A History of Anarchism, PM Press, 2009.

[19] Cataldo, M., Herbsleb, J., and Carley, K., "SocioTechnical Congruence: A Framework for Assessing the Impact of Technical and Work Dependencies on Software Development Productivity", international symposium on Empirical software engineering and measurement, 2008, pp. 2-11.

[20] Hylving, L., and Schultze, U., "Evolving the Modular Layered Architecture in Digital Innovation: The Case of the Car's Instrument Cluster", International Conference on Information Systems 2013

[21] Boland, R.J., Lyytinen, K., and Yoo, Y., "Wakes of Innovation in Project Networks: The Case of Digital 3-D Representations in Architecture, Engineering, and Construction", Organization Science, 18(4), 2007, pp. 631647.

[22] Yoo, Y., "Computing in Everyday Life: A Call for Research on Experiential Computing", Management Information Systems Quarterly, 34(2), 2010, pp. 213-231.

[23] King, J., L., and Lyytinen, K., "Automotive Informatics: Information Technology and Enterprise Transformation in the Automobile Industry", in (Kahin, B., and Wycoff, A., 'eds.'): Information Technology and Enterprise Transformation, MIT Press, Cambridge, MA, 2004

[24] [Andersson, M., Lindgren, R., and Henfridsson, O., "Architectural Knowledge in Inter-Organizational It Innovation", Journal of Strategic Information Systems, 17(2008, pp. 19-38.

[25] Van De Ven, A.H., "Running in Packs to Develop Knowledge-Intensive Technologies", MIS quarterly, 29(2), 2005, pp. 365-378.

[26] Tuomi, I., Networks of Innovation, Oxford University Press Oxford, 2002.

[27] Crowston, K., Wei, K., Howison, J., and Wiggins, A., "Free/Libre Open-Source Software Development: What We Know and What We Do Not Know", ACM Computing Surveys (CSUR), 44(2), 2012, pp. 7.
[28] Rudmark, D., and Ghazawneh, A., "Third-Party Development for Multi-Contextual Services: On the Mechanisms of Control", in (Editor, 'ed.'^'eds.'): Book Third-Party Development for Multi-Contextual Services: On the Mechanisms of Control, 2011

[29] Woodcock, G., Anarchism, Springer, 1989.

[30] Dimaggio, P.J., "Interest and Agency in Institutional Theory", in (Zucker, L.G., 'ed.' Institutional Patterns and Organizations: Culture and Environment, Ballinger, Cambridge, MA, 1988

[31] Greenwood, R., and Suddaby, R., "Institutional Entrepreneurship in Mature Fields: The Big Five Accounting Firms", Academy of Management journal, 49(1), 2006, pp. 27-48.

[32] Eisenhardt, K.M., "Building Theories from Case Study Research", Academy of Management Review, 14(4), 1989, pp. 532-550.

[33] Meredith, J., "Building Operations Management Theory through Case and Field Research", Journal of operations management, 16(4), 1998, pp. 441-454.

[34] Dimaggio, P., and Powell, W., "The Iron Cage Revisited: Institutional Isomorphism and Collective Rationality in Organizational Fields", American sociological review, 48(2), 1983, pp. 147-160.

[35] "anarchy". 2016. In Merriam-Webster.com. Retrieved May 8, 2016, from http://www.merriamwebster.com/dictionary/anarchy

[36] Tuertscher, P., Garud, R., \& Kumaraswamy, A. (2014). Justification and interlaced knowledge at ATLAS, CERN. Organization Science, 25(6), 1579-1608

[37] Braun, Virginia, and Victoria Clarke. "Using thematic analysis in psychology."Qualitative research in psychology 3.2 (2006): 77-101. 